\title{
CONSTRUYENDO EL CALEIDOSCOPIO: LA HISTORIA SUBNACIONAL
}

\author{
Aurora Gómez-Galvarriato Freer \\ El Colegio de México
}

El tiempo y el lugar son las dos coordenadas de la historia. tiempos clásicos como sus dos ojos, pero como el tiempo ha jugado el papel principal, la corografía se ha identificado como su ojo izquierdo. ${ }^{2}$ Mientras que los historiadores han tenido absoluta soberanía sobre la dimensión temporal del ser humano, su dimensión espacial es disputada por la geografía. Sin embargo, como señalaba Bernardo García Martínez, puesto que la realidad humana comprende las dos dimensiones, ambas "deberían estar tan estrechamente unidas en el conocimiento como lo están en la realidad; por eso, algún día debiera la historia conquistar ese territorio de la dimensión espacial". 3

El desinterés por el espacio se ha debido en gran medida al predominio de las historias universales y nacionales, pues en ellas el espacio está predeterminado y el historiador no tiene que ocuparse mucho de él. Como señalaba Luis González, "el espacio

\footnotetext{
${ }^{1}$ Geografía regional; su nombre viene del griego chóros (un trozo de tierra ocupada por una persona o cosa).

2 Withers, "Place and the 'Spatial Turn'”, p. 639.

3 García Martínez, "Consideraciones corográficas", p. 7.
} 
geográfico de la historia universal es obra de la naturaleza, es la bola de billar denominada mundo. El espacio de la historia continental no es menos inhumano. El espacio de la historia nacional lo determinan convenios y guerras conforme a vagas razones de Estado". ${ }^{4}$ En contraste, "el espacio de la historia local tiene límites poco precisos y muy cambiantes, oriundos del sentimiento y de la acción”, por lo que el historiador ha de definirlo y eso lo obliga a profundizar en el mismo. Mientras que "en la historia crítica lo básico es el tiempo, la oposición entre unas épocas y otras. En la historia local lo importante es el espacio". ${ }^{5}$

El espacio es un concepto fundamental en la geografía humana, por lo que los geógrafos son quienes más han reflexionado sobre el mismo. El espacio, nos dicen Hiernaux y Lindon, se puede entender de varias maneras. Una de ellas es verlo como espacio continente o receptáculo, es decir, como "un mero soporte o sustrato sobre el cual se localizan elementos y relaciones". ${ }^{6}$ Sin embargo, también puede entenderse como espacio reflejo, esto es como "un espejo de la sociedad y las relaciones sociales", 7 en el que todo cambio social es reflejado, o bien como espacio estructura o bien como instancia integrante de la totalidad social que es subordinante-subordinado, productor y producido. La antropología también ha problematizado el espacio y reflexionado sobre el concepto de región. Como explica Guillermo de la Peña, para la antropología social es claro, como varios de sus estudios han mostrado empíricamente, "que el concepto de espacio es socialmente creado, porque es socialmente vivido". ${ }^{8}$ La región, dice De la Peña, "no es simplemente algo que está allí, sino un espacio privilegiado de investigación

4 GonzÁlez y GonzÁlez, “Microhistoria para Multiméxico” (82), 1971, pp. 225-241, esp. p. 226.

${ }^{5}$ González y González, "Hacia una teoría de la microhistoria”, p. 7.

${ }^{6}$ Hiernaux y Lindon, "El concepto de espacio", p. 90.

7 Hiernaux y Lindon, "El concepto de espacio", p. 91.

8 PeÑa, "Los estudios regionales", p. 46. 
que se construye tanto por el observador como por los sujetos que viven el espacio". ${ }^{9}$ Bajo estas perspectivas el espacio, como función de una sociedad, cambia a lo largo del tiempo y es un sujeto histórico.

Los historiadores somos poco proclives al análisis teórico, por lo que hemos discernido menos sobre estas cuestiones. Esto obedece, como señala Eric Van Young, a que "la mayoría de nosotros piensa que ya sabe lo que es una región: es el área que estamos estudiando en este momento [...]. Las regiones son como el amor, son difíciles de describir, pero las conocemos cuando las vemos". ${ }^{10}$ Sin embargo, consideraba que la falta de claridad sobre el concepto de región generaba el problema "de estar operando con un concepto complejo antes de definirlo". ${ }^{11} \mathrm{Su}$ propuesta es entenderla como "un espacio geográfico con una frontera que lo activa, la cual estaría determinada por el alcance efectivo de algún sistema cuyas partes interactúan más entre sí que con los sistemas externos”. ${ }^{2}$ Para Van Young, en el trabajo historiográfico "las regiones son hipótesis por demostrar". ${ }^{13}$

Juan Pedro Viqueira explica que las regiones pueden concebirse a partir de distintas variables. Una forma usual de hacerlo es utilizando elementos geográficos u otros criterios "naturales", como la distribución de personas hablantes de la misma lengua. Otro es delimitarlas por medio de "fenómenos sociales totales” o por algún elemento de especial relevancia de la realidad (actividades económicas, redes de comercio y de intercambio de información, dependencia de determinados centros urbanos, etc.). Otro enfoque es identificarlas como espacios vividos, es decir, por la conciencia que las personas tienen de su espacio social. Esta perspectiva, común en la antropología, resulta de difícil

\footnotetext{
9 PEÑA, “La región: visiones antropológicas”, p. 13.

10 VAN YounG, "Haciendo historia regional", p. 255.

11 VAn Young, “Haciendo historia regional”, p. 257.

12 VAN Young, “Haciendo historia regional”, pp. 257-258.

13 VAN Young, "Haciendo historia regional”, p. 257.
} 
manejo para los historiadores debido a la escasez de testimonios al respecto. Para Viqueira, cualquier definición de región radica a fin de cuentas "en la voluntad -rara vez consciente- de explicar la complejidad social a partir de un solo factor o de un pequeño conjunto de éstos" ${ }^{14}$ Sin embargo, ninguna puede ser desechada en forma definitiva pues "el estudio de la distribución espacial de cualquier variable natural o humana puede enriquecer en mayor o menor grado nuestro conocimiento de la realidad". ${ }^{15}$ Advierte, sin embargo, que hay que cuidarse de extrapolar lo observado en cierto nivel de la sociedad al conjunto de ésta. Recomienda a los historiadores superar los límites que implica cada una de estas definiciones y buscar una visión más amplia de la realidad social, que "pasa necesariamente por la reconstrucción de las historias de las distribuciones espaciales de determinadas variables sociales y los desfases existentes entre unas y otras". ${ }^{16}$

Después de años de estudio y reflexión como un historiador comprometido con la geografía, Bernardo García Martínez propuso en su libro Las regiones de México. Breviario geográfico e histórico, una división espacial de México en regiones, no determinada por límites políticos o jurisdiccionales, sino por la formación histórica, social, económica y cultural de los grupos humanos sobre ciertos medios ambientes, así como por la percepción de pertenencia de esos habitantes a una región específica. Explica que "las regiones son un producto histórico enlazado con un medio físico. Son un terreno en que se expresan con gran intimidad las relaciones entre la geografía y la historia, o para decirlo de otra manera, entre el espacio y el tiempo". ${ }^{17}$ Para Bernardo García, la cuestión clave para definir una región, o tejido espacial, son los movimientos e intercambios humanos a lo largo del tiempo. Se trata de un sistema funcional esencialmente

\footnotetext{
14 Viqueira, “Regiones naturales”, p. 115.

15 Viqueira, “Regiones naturales”, p. 115.

16 Viqueira, “Regiones naturales”, p. 115.

17 García Martínez, Las regiones de México, p. 12.
} 
vivo en el que existen "flujos de gente, productos, información y decisiones, así como una red de enlaces que los permitan, además de puntos o nodos que amarren todo ello conforme a ciertas jerarquías y dentro de un área definible”. ${ }^{18}$ Bernardo García define la región como: “un espacio funcional y dinámico que alberga relaciones, intercambios e identidades culturales integradas históricamente y cuya individualidad es percibida por quienes participan en ella [...]. Cada región es un ámbito en el cual opera un sistema en constante movimiento". ${ }^{19}$

Otro problema muy debatido ha sido lo que ha de entenderse por historia regional y sus diferencias con otro tipo de historias subnacionales. Hace casi dos décadas Manuel Miño Grijalva se cuestionaba: ¿existe la historia regional? Hacerse esta pregunta surgía de las dificultades que observaba en el uso de este término, en que "los historiadores la han concebido más con los contenidos geográficos y naturales que con los procesos sociales, o simplemente, se da por supuesto, que cualquier estudio, al referirse a una sociedad provincial ya, de por sí, es historia regional". ${ }^{20}$ La región, explicaba, como cualquier otro ámbito espacial, constituye simplemente el marco físico donde se desarrollan los procesos históricos y su delimitación se realiza con el auxilio de otras ciencias sociales. Por tanto, consideraba que no podemos pensar en ella como una disciplina dotada de unidad conceptual y de un cuerpo metodológico o analítico específico. ${ }^{21}$ A Miño le preocupaba también que no se diferenciaran los distintos tipos de historias subnacionales. ${ }^{22}$ Como señala Gilbert Joseph, los historiadores “rara vez distinguen entre historia regional y local, y la mayoría emplea el concepto

\footnotetext{
18 García Martínez, Las regiones de México, p. 28.

19 García Martínez, Las regiones de México, p. 29.

20 Miño Grijalva, “¿Existe la historia regional?” (204), 2002, pp. 867-897, esp. p. 867.

21 Miño Grijalva, “¿Existe la historia regional?” (204), 2002, p. 893.

22 Miño Grijalva, “¿Existe la historia regional?” (204), 2002, pp. 867-868.
} 
de "historia regional" como una forma conveniente de referirse a toda la historia subnacional". ${ }^{23}$

Luis González consideraba que la historia regional y la microhistoria eran distintas no sólo en cuanto a su ámbito espacial, sino también por su metodología. La microhistoria, o historia pueblerina, parroquial, de la patria chica, o de campanario, como solía llamarla Luis González de manera intercambiable, debía ser, ante todo, el relato verdadero, concreto y cualitativo del pretérito de la vida diaria del hombre común. ${ }^{24}$ La microhistoria, como "historia matria", se aboca al "mundo pequeño, femenino, sentimental de la madre [...] es decir, la familia, el terruño", al estudio de comunidades donde predominan los lazos de sangre y el conocimiento mutuo entre las personas. Su objetivo era fundamentalmente la "comprensión de los actores" mediante un análisis más subjetivo que analítico y que generalmente no se restringía a un tema. ${ }^{25}$ "La microhistoria", decía, "es la menos ciencia y la más humana de las ciencias del hombre", ${ }^{26}$ si bien, siempre insistió que exigía, como ninguna otra, "la aplicación de todas las técnicas heurísticas, críticas, interpretativas, etiológicas, arquitectónicas y de estilo". ${ }^{27}$ En contraste, la historia regional se ocupaba de "la comunidad mediana donde son particularmente importantes los lazos económicos” y ésta se relacionaba más con las ciencias sociales.

A lo largo del tiempo, estas diferencias han sido desbordadas. Así, para Carlos Martínez Assad, la historia regional

[...] busca destacar la otra historia, la de lo cotidiano, la del conflicto que confronta a actores sociales en un espacio que puede ser más

23 JosEph, “La nueva historiografía regional”, p. 54.

24 Arias, “Luis González. Microhistoria e historia regional”, p. 181.

25 GonzÁlez y GonZÁlez, “Historia regional en sentido riguroso”, p. 194.

26 GonzÁlez y GonzÁlez, “Terruño, microhistoria y ciencias sociales”, pp. 30 y 31 .

27 González y González, Pueblo en vilo, p. 16. 
o menos grande, pero que se refiere siempre a la búsqueda de lo específico. Ese espacio que no es el de las fronteras establecidas, que no está señalada por el cauce de los ríos, sino por los movimientos sociales y acontecimientos políticos que lo definen. ${ }^{28}$

Un elemento básico de la historia regional en el que concuerdan los historiadores es que al practicarla es necesario definir los linderos del espacio a estudiar, como parte del proceso de investigación. Bajo esta perspectiva, las historias que parten de un espacio predeterminado, definido por delimitaciones político-administrativas, no podrían considerarse historias regionales. Muchos de estos trabajos, señala Antonio Ibarra, son "una suerte de "historia regional institucionalizada" que ha respondido al propósito de hacer una "estatografía” de cada entidad federativa, a contrapunto de la historia nacional y como respuesta al “centralismo". ${ }^{29}$ Debido a que los archivos se organizan considerando una unidad política o administrativa, las fuentes delimitan el espacio de estudio, lo que da lugar "a regiones que se igualan con entidades federativas o ciudades, aun cuando no necesariamente el hecho estudiado la llene o incluso la rebase". ${ }^{30}$ Sin embargo, detrás de muchos títulos que aluden a estados o provincias, se encuentran verdaderas historias regionales.

Además, las historias regionales (o subnacionales) se distinguen también por los distintos objetivos que persiguen. ${ }^{31}$ Algunas de ellas analizan los fenómenos generales ocurridos en regiones concretas, como herramienta para comprender una historia más amplia. Estas investigaciones buscan identificar las peculiaridades regionales "para explicar el proceso histórico de la sociedad colonial o nacional en su conjunto, y moderar así las

28 Martínez Assad, “Eslabones” (200), 2001, pp. 921-943, esp. p. 942.

29 Ibarra, “Un debate suspendido” (205), 2002, pp. 241-259, esp. p. 243.

30 Martínez Assad, “Eslabones” (200), 2001, p. 942.

31 González Martínez, Alvarado y el mundo del agua, p. 32. 
imprecisas o inexactas generalizaciones que se suelen hacer de manera frecuente". ${ }^{32}$ Varias de ellas son estudios de caso "en los cuales una cantidad de información local, extraordinariamente rica y diversa ilumina una serie de problemas históricos mayores que les permite poner a prueba la sabiduría convencional y, con cierta frecuencia, replantearla". ${ }^{33}$ Para investigadores como Antonio Ibarra, este enfoque permite situar la investigación en una "escala de medición regional" en una tentativa de formalizar un problema que trasciende a lo estrictamente local. ${ }^{34}$

En contraste, para otros historiadores, como Luis González, la aportación de la historia local va más allá “de sumar las historiografías parroquiales o al menos las de las parroquias más representativas de cada región" para "llegar a una verdadera historia nacional", como si se tratara de las piezas de un mosaico. "Es innegable”, decía, "que conocer los pedazos de alguna manera sirve para el conocimiento del conjunto", pues "la historia de algunas naciones como la mexicana son inenarrables si no se alude a las muchas nacioncitas que la conforman". ${ }^{35}$ Sin embargo, juzgaba que su mayor contribución era que "la vida de las comunidades pequeñas [...] aporta experiencias humanas ejemplares para cualquier hombre, tanto porque los campesinos tiene cosas que enseñar", como porque "la historiografía microscópica [...] contiene más verdad que la telescópica”, al permitir una mayor aproximación a la realidad humana. ${ }^{36}$

La delimitación de lo que es o no es historia regional no es sencilla. A lo largo de los años, varios historiadores que han participado como jueces del premio en historia regional "Atanasio G. Saravia”, llegaron al siguiente consenso:

\footnotetext{
32 MiÑo Grijalva, “¿Existe la historia regional?” (204), 2002, p. 883.

33 Joseph, "La nueva historiografía”, p. 54.

34 Ibarra, "Un debate suspendido” (205), 2002, p. 244.

35 González y González, Pueblo en vilo, p. 15.

36 González y González, Pueblo en vilo, p. 15.
} 
Se entiende por historia regional el estudio de los hechos, los procesos, las transformaciones y las continuidades históricas de un espacio geográfico, social y cultural que comparte características que lo hacen único y diferente de otras áreas. No se entiende por historia regional el análisis de lo sucedido en una localidad que no tiene repercusiones en el entorno regional, por ejemplo: la biografía de un personaje, de una institución, de un edificio, desarrollo de una lengua, entre otros casos. ${ }^{37}$

Como señala Antonio Ibarra, "la historia 'regional' se convirtió en un río de varias aguas que aun hoy resulta difícil de medir en su caudal y distinguir en sus corrientes cristalinas, entre la turbulencia de tanta monografía 'regional' que la enturbia”. ${ }^{38}$ Dada la dificultad que existe en identificar la historia regional y los distintos significados que este término ha tenido a lo largo del tiempo, en este ensayo se optó por apegarse a la categoría general de historia subnacional, con el objetivo de explorar su evolución a la luz de la revista Historia Mexicana durante los últimos 70 años. En las siguientes páginas presentaremos en primer lugar una perspectiva general sobre cómo se dio este proceso en el ámbito internacional, así como en nuestro país. Estudiaremos después su evolución en las páginas de la revista Historia Mexicana.

\section{LA HISTORIA DE LA HISTORIA SUBNACIONAL EN LA HISTORIOGRAFÍA}

La elección del espacio a estudiar por los historiadores ha cambiado a lo largo del tiempo de forma diversa en distintos lugares.

37 "Convocatoria del Premio Atanasio G. Saravia de Historia Regional Mexicana 2018”, http://fomentoculturalbanamex.org/noticias/convocatoria-delpremio-atanasio-g-saravia-de-historia-regional-mexicana-2018/ Consultado el 2 de octubre de 2020.

38 Ibarra, "Un debate suspendido" (205), 2002, p. 244. 
A partir del triunfo del Estado-nación durante el siglo xix, la nación se convirtió en el espacio más comúnmente estudiado por los historiadores, en gran medida resultado de las propias necesidades políticas, que requerían de historias patrias para la propia construcción del Estado-nación. "Los historiadores", escribió Eugen Weber, "eran la clerecía del siglo xix porque les tocó a ellos reescribir los mitos fundacionales; y la historia era la teología del siglo xix porque proveía a las sociedades recién liberadas de las ataduras de la costumbre y el hábito con un nuevo anclaje en un pasado redescubierto-o inventado". ${ }^{39}$ Los historiadores en distintos países escribieron sobre las fundaciones de las naciones, su pasado y la coherencia de la unidad nacional. "La devaluación de las regiones y sus pasados en el siglo XIX, por tanto, emergió naturalmente junto con el triunfo de las historiografías nacionales", ${ }^{40}$ escribe Celia Applegate al reflexionar sobre la historia regional europea. El estudio de las provincias y localidades no desapareció, pero se subordinó al proyecto de la historia nacional y fue desarrollado principalmente por aficionados.

Sin embargo, la nación como el espacio a estudiar les fue resultando una camisa de fuerza a muchos historiadores. Esto les resultó así, en primer lugar, a quienes se especializaban en periodos previos a la construcción de los Estados-nación. En Francia, Marc Bloch y Lucien Febvre incursionaron en los ámbitos regionales y locales y contextualizaron los procesos históricos en términos de la "geografía humana" y el medio ambiente. La escuela de los Annales insistió en la necesidad de una "geografía histórica", en la búsqueda del arraigo espacial de los acontecimientos, "de los fundamentos naturales ofrecidos a las fuerzas productivas desarrolladas por el hombre". ${ }^{41}$ Por su parte, en los

\footnotetext{
39 Weber, My France: Poltics, Culture, Myth, p. 23.

40 Applegate, “A Europe of Regions", p. 1159.

${ }^{41}$ VILAR, "Sobre los fundamentos de las estructuras nacionales", p. 13.
} 
años de entreguerras, en Alemania surgió la historia étnica (Volksgeschichte) e historiadores como Hermann Aubin o Rudolf Kötzschke dieron nuevo vigor a la tradición del Landesgeschichte mediante la introducción de nuevas metodologías y análisis comparativos.

Después de la segunda guerra mundial, por varias décadas, el discurso del historiador profesional fue la teoría de la modernización. Esta estructura conceptual jugó contra el papel de las regiones, pues suponía que éstas eventualmente desaparecerían por medio de un proceso de homogeneización. Sin embargo, durante la década de 1970, perdieron fuerza estas teorías y los historiadores dejaron de hacer supuestos axiomáticos. En este proceso tuvieron gran influencia los italianos Carlo Ginzburg y Giovanni Levi, que dieron gran impulso a la microhistoria. A partir de entonces, comenzaron a publicarse en Europa gran cantidad de estudios históricos regionales o locales.

En contraste, en Estados Unidos, la historia nacional fue vista desde temprano como un proceso secuencial de desarrollo regional. En 1948, William Heseltine escribía que "como resultado del hecho que la vida americana se ha organizado desde una base regional, surgen implicaciones para el estudio y escritura de la historia americana", y consideraba que ésta no podría escribirse sino hasta que las historias de sus distintas regiones hubieran sido estudiadas, evaluadas e integradas. ${ }^{42}$ La forma como se construyó dicha nación y la importancia de sus diferencias regionales para explicar la política nacional dieron a las regiones una particular importancia en la historiografía. Asimismo, el temprano surgimiento de universidades en sus distintos puntos geográficos contribuyó al desarrollo de la historia regional, que se incorporó a la instrucción de las universidades en ese país desde la última década del siglo xIx. La historia del Oeste se comenzó a enseñar en la Universidad de Wisconsin en 1895.

42 Heseltine, “The Value of Regional History”, p. 12. 
$\mathrm{Al}$ año siguiente, la Universidad Johns Hopkins ofreció el primer curso sobre la historia del Sur ofrecido fuera del Sur; en la Universidad de Chicago, desde 1906.43

En México, como explica Luis González, la tradición de la historia local se remonta a la época prehispánica, pues en ese entonces no existía el concepto de historia general. Después, durante la época virreinal, aunque no fue la especie predominante, ésta se diversificó y tuvo mejores fuentes de información. La corona alentó la realización de relaciones histórico-geográficas, florecieron las crónicas conventuales y en el siglo XviII cundió el interés por la historia urbana. Después de la independencia, a pesar del nacionalismo, se patrocinaron noticias geográficas y estadísticas de distintas localidades. Luego, hacia 1833, México sale de "una etapa de euforia nacionalista y se inscribe en un período ásperamente localista", en el que aparecieron diversos libros sobre distintas ciudades. En cambio, durante la segunda mitad del siglo xIX, como resultado de las invasiones extranjeras, se robusteció el nacionalismo, se propició la historia nacional y se "le hizo el feo a las historias estatal y local". ${ }^{44}$ No obstante, los gobiernos locales patrocinaron durante el porfiriato más de 100 libros históricos subnacionales, los más de historia de los estados, pero también algunos de índole local. A partir de la revolución mexicana, entre 1910 y 1940, el nacionalismo resurgió, pero muchos defendieron la tesis de que se podía ser localista sin dejar de ser patriota. De esta forma, entre 1911 y 1940 se publicaron 148 libros de historia regional y local, de los cuales $57 \%$ eran historia de los estados. Las publicaciones de historias subnacionales aumentaron de 1941 a 1970, llegando a alrededor de 300, entre las que predominaron las historias locales, con $60 \%$ del total..$^{45}$

43 Аввотт, "United States Regional History”, p. 199.

44 González y González, “Microhistoria para Multiméxico” (82), 1971, pp. 229-231.

45 González y González, “Microhistoria para Multiméxico” (82), 1971, pp. 229-231. 
Sin embargo, muchas de estas obras fueron realizadas por amateurs, sin formación historiográfica, sin auxilios institucionales y aislados del mundo intelectual. El estudio profesional de la historia de los espacios subnacionales demoró en llegar, a pesar de que la disciplina se fue profesionalizando, pues "estaban de moda la historia crítica y las filosofías de la historia que buscaban 'las fuerzas impersonales' que empujan a la humanidad a partir de las cuales de abstraían leyes de la historia". ${ }^{46}$ Gradualmente, estas corrientes perdieron importancia y la historia local y regional fue ganando terreno.

Un actor fundamental en este proceso fue Luis González. En su contribución a La vida social de la Historia moderna de México, publicada en 1956, hizo un ejercicio complejo de historia regional en el que subrayó la diversidad socioespacial y cultural del país y mostró la necesidad de pensar en México en su diversidad y pluralidad. ${ }^{47}$ Pero la obra que contribuyó de forma crucial a despertar el interés por la historia local fue su publicación en 1968 de Pueblo en Vilo. Microbistoria de San José de Gracia. En ella Luis González mostró que cualquier comunidad ofrece una riqueza de materiales al historiador y que este trabajo podía emprenderse de forma rigurosa para producir una obra relevante. A partir de entonces, por medio de diversas publicaciones y su labor docente, don Luis realizó una campaña en favor de la historia local, que vislumbraba tenía grandes potenciales en el país:

Quizá por ser un país en vías de desarrollo, quizá por mantenerse disímbolo a pesar de los esfuerzos igualadores de la modernización, quizá porque su historia nacional es sólo piel y sus entrañas son particularistas, quizá por la supervivencia de los amores al terruño, México es particularmente proclive a la historiografía menuda. El

46 ARIAS, "Luis González. Microhistoria e historia regional”, p. 178.

47 Arias, "Luis González. Microhistoria e historia regional”, p. 184. 
camino natural de la ciencia histórica mexicana es localista. Los otros caminos han sido impuestos muchas veces por el poder, la imitación extranjerizante, la moda y la pedantería universitaria. ${ }^{48}$

La impronta de Luis González confluyó con un momento en el que el país vivía una crisis social y política en diferentes ciudades y regiones y en el que, como señala Leticia Reina, "todos apuntaban a cuestionar las políticas estatales o centralistas que poco atendían a las necesidades de cambio de la provincia y de los diferentes sectores sociales que conformaban la sociedad nacional". ${ }^{49}$ Las investigaciones regionales, ubicadas en lo que se ha definido como el revisionismo histórico, señala Carlos Martínez Assad, "pusieron el dedo en la llaga porque demostraron que la historia de México no fue un proceso monolítico ni homogéneo" y enfatizaron “más las diferencias que las coincidencias". ${ }^{50}$ Además, entre 1950 y 1970 se fueron abriendo departamentos de historia en las universidades de la mayor parte de las entidades federativas.

De esta forma, durante la década de 1970 inició el auge de los estudios históricos subnacionales. En agosto de 1972 tuvo lugar el Primer Encuentro de Historiadores de Provincia y en él se constituyó la Asociación Mexicana de Historia Regional A. C. ${ }^{51}$ Luego, en 1979, se creó El Colegio de Michoacán, que organizó ese mismo año el Primer Congreso de Historia Regional. A esto se aunó una gradual y lenta mejora de los archivos y bibliotecas estatales, municipales y eclesiásticos, en parte propiciada por los gobiernos, pero también por la labor de las universidades y particulares e instituciones como

48 González y González, “Microhistoria para Multiméxico” (82), 1971, p. 235.

49 ReInA, "Historia regional e historia nacional”, p. 134.

50 Martínez Assad, “Eslabones” (200), 2001, p. 921.

51 Velázquez, “Tercer Encuentro de Historiadores de Provincia” (102), 1976, pp. 339-340. 
ADABI de México, ${ }^{52}$ que han contribuido significativamente en esta labor.

Durante la siguiente década se organizaron varios seminarios y congresos que promovieron la historia regional. A partir de ellos, en 1990 se constituyó la Sociedad Nacional de Estudios Regionales A. C., conformada por varios historiadores y científicos sociales de diferentes estados que inició al año siguiente la publicación de Eslabones, Revista semestral de estudios regionales. Su primer comité editorial estuvo conformado por destacados historiadores e intelectuales. ${ }^{53}$

\section{LA REVISTA HISTORIA MEXICANA}

\section{Y LA HISTORIA SUBNACIONAL}

Hacia finales del siglo xx, la perspectiva subnacional en la historiografía mexicana había llegado a una madurez, pero el trayecto no fue sencillo, como lo muestran las páginas de la revista Historia Mexicana a lo largo de sus 70 años de existencia. La revista no sólo fue un foro de este proceso, sino que contribuyó en él de forma deliberada.

Durante los primeros años de Historia Mexicana prácticamente todos sus artículos versaban sobre la historia nacional. Sin embargo, desde su primer número ésta se preocupó por el desarrollo historiográfico de los distintos estados del país, inaugurando una serie de artículos que se llamaron "La historia y sus instrumentos", que abordaba la situación de la ciencia histórica -sus posibilidades y sus logros- en diversas entidades federativas. En el primero de dichos artículos, Miguel de la Mora L. y Moisés González Navarro expresaban:

52 La Asociación de Apoyo al Desarrollo de Archivos y Bibliotecas de México A. C., fundada en 2004, es una asociación civil que impulsa proyectos enfocados al rescate y conservación del patrimonio documental del país.

53 Martínez Assad, "Eslabones” (200), 2001, pp. 921-943. 
La dirección de esta Revista ha pensado que es útil presentar en una serie de artículos la situación de los trabajos historiográficos en los diversos estados de la República, pues considera que existe una importante labor que se realiza en la provincia, la cual es preciso dar a conocer a los investigadores de esta disciplina, a fin de poder hacer un balance de dichos estudios con la mira de que de él salgan sugestiones para conectar estas actividades en toda la República. ${ }^{54}$

Esta iniciativa se debió, sin duda, al interés por despejar la brecha y estimular el desarrollo de la historia subnacional, pues como expresó Daniel Cosío Villegas en la tercera llamada particular con que inicia el volumen de La vida social, si bien su objetivo era hacer una historia verdaderamente nacional y no sólo "capitalina”, encontraron que la historia regional no estaba aún trabajada. Pero el mayor obstáculo que enfrentaron fue que no había en México “suficiente información sobre las provincias, ni documentos ni publicaciones periódicas ni libros siquiera" ${ }^{55}$

Entre 1951 y 1975 se publicaron ocho artículos de esta serie, que informaron sobre la situación y potencialidades del quehacer histórico en los estados de Jalisco, Nuevo León, Michoacán, Oaxaca, Durango, Querétaro y Puebla y la ciudad de Xalapa. Éstos evidencian los enormes retos que limitaban el desarrollo profesional de la historia subnacional. Durante las décadas de 1950 y 1960, no era común que se impartiera en las escuelas, en ningún grado, la historia particular de los estados. ${ }^{56}$ Solamente en Nuevo León y Querétaro se enseñaba a los niños y jóvenes la historia del estado. ${ }^{57}$ Dicha situación fue en parte

\footnotetext{
54 Mora y González Navarro, “Jalisco: la historia y sus instrumentos” (1), 1951, pp. 143-163, esp. p. 143.

55 Cosío Villegas, Historia moderna, p. xxxiii.

56 Mora y González Navarro, “Jalisco: la historia y sus instrumentos” (1), 1951, p. 146.

57 Cavazos Garza, “Nuevo León: la historia y sus instrumentos” (3), 1952, pp. 494-515, esp. p. 494; GARcía MARTíneZ y LiRa GonzÁlez, “Querétaro:
} 
el resultado, como explica Jorge Fernando Iturribarría, de la federalización de la educación pública, que constreñía a seguir el programa de la Secretaría de Educación Pública. De esta forma, por ejemplo, en el Instituto de Ciencias y Artes de Oaxaca, la clase de historia del estado, que había sido obligatoria desde 1930, quedó suprimida a partir de $1948 .^{58}$

Un obstáculo mayor fue también el lento surgimiento de escuelas o departamentos de historia en las universidades estatales. Hacia 1950, éstas no existían en las universidades de Jalisco, Michoacán, Oaxaca, Durango y Querétaro, pero la situación comenzó a cambiar a partir de esa década. En 1951, en Jalisco se decía que la Universidad de Guadalajara pronto fundaría un Centro de Estudios Históricos, el cual "prestaría valiosos servicios al estudio de la historiografía jalisciense pues en él se formarían los futuros investigadores de la misma". ${ }^{59}$ Hacia 1952, la Universidad de Nuevo León recientemente había creado la Facultad de Filosofía y Letras, en donde se impartían cursos de historia. ${ }^{60}$ Por su parte, en Xalapa la escuela de historia se creó en 1957, dependiente de la Facultad de Filosofía y Letras de la Universidad Veracruzana. ${ }^{61}$ En Durango en 1960 se fundó la cátedra de historia de Durango en la Universidad Juárez. ${ }^{62}$ La Universidad de Puebla creó

\footnotetext{
la historia y sus instrumentos" (70), 1968, pp. 286-292, esp. p. 287.

58 ItUrribarría, “Oaxaca: la historia y sus instrumentos" (7), 1953, pp. 459476, esp. p. 461.

59 Mora y González Navarro, “Jalisco: la historia y sus instrumentos” (1), 1951, p. 146.

60 Cavazos Garza, "Nuevo León: la historia y sus instrumentos” (3), 1952, p. 495.

${ }^{61}$ Sudo y De los Reyes, "Xalapa: la historia y sus instrumentos (96), 1975, pp. 607-621, esp. p. 611. p. 608.

62 Gallegos, "Durango: la historia y sus instrumentos" (42), 1961, pp. 314320, esp. p. 314.
} 
su colegio de Puebla en 1966 y el Instituto Poblano de Antropología e Historia comenzaba a interesarse por la historia regional de Puebla-Tlaxcala. ${ }^{63}$

La ausencia de espacios universitarios para la historia impedía que la disciplina contara con un sustento económico y profesional. El profesor José Cornejo Franco, impulsor de la investigación historiográfica en Jalisco, explicaba que en el estado los cultivadores de la historia lo hacían "por afición y no como profesionales" ${ }^{64}$ En el mismo sentido, el profesor Manuel Zárate Aquino, de Oaxaca, expresaba que "la investigación histórica, realizada por el solo gusto de investigar, es factible cuando el investigador no tiene necesidad de dedicarse a otras actividades que le permitan sufragar sus gastos más apremiantes". Estimaba que para el desarrollo de la disciplina en Oaxaca era necesario colocar a los investigadores "en condiciones tales que les permitan hacer investigación histórica seria, intensa, sistemática y exhaustiva" y "lograr la publicación de los resultados de su investigación". ${ }^{65}$

Otro problema fundamental que enfrentaba el quehacer histórico en los estados era el difícil acceso a fuentes documentales. Señalaba Luis González:

¿Quién no reconoce la pobreza y mal funcionamiento de las bibliotecas públicas? ¿Quién ignora el desorden de nuestros archivos? La mayoría de las fuentes de la historia local no ha sido recogida aun en los repositorios públicos. Muchas han sido sustraídas por bibliómanos y maniacos de los papeles viejos y se guardan bajo siete llaves en arcones privadísimos. Otras han sido entregadas a la

63 Bazant, "Puebla: la historia y sus instrumentos" (75), 1970, pp. 432-437, p. 435.

${ }^{64}$ Mora y González Navarro, "Jalisco: la historia y sus instrumentos" (1), 1951, p. 146.

${ }^{65}$ ItUrRibarría, “Oaxaca: la historia y sus instrumentos” (7), 1953, p. 475. 
acción destructiva de la humedad, o del aire, o del fuego o vendidas para servir de papel de envoltura. ${ }^{66}$

Los artículos de "la historia y sus instrumentos" evidencian el profundo atraso en el que se encontraban los archivos y bibliotecas estatales. En Guadalajara, por ejemplo, hacia 1951, en el Archivo de Instrumentos Públicos, que comprendía los ramos de Tierras y Aguas, Repartimientos de Indios y Protocolos de Notarías, sólo se permitía la consulta del primero de esos ramos, y para investigar en los otros dos, era necesario recabar en permiso de la Secretaría de Gobierno. Además, los archivos se encontraban sin catalogar y en desorden. El artículo sobre Michoacán narra la trágica historia de muchos archivos de los que varios valiosos fondos fueron destruidos o desaparecidos. ${ }^{67}$ Hacia 1968 , los profesores Bernardo García y Andrés Lira señalaban que, en Querétaro, los archivos eran magníficos, pero "desgraciadamente poco accesibles, sea a causa del abandono y deterioro en que se encuentran, sea debido a la mala administración que los agobia" ${ }^{68}$

Pero los autores de "la historia y sus instrumentos" no se dejaban derrotar por estos problemas. Los artículos promovían el enorme potencial que existía para el trabajo histórico de cada estado y exploraban la gran cantidad de fuentes disponibles para realizarlo, detallando con la mayor precisión posible su ámbito, periodo y localización. Los artículos planteaban que la historia local, estatal o regional era un terreno fértil para la investigación histórica. ${ }^{69}$

66 González y González, “Microhistoria para Multiméxico” (82), 1971, pp. 225-241, esp. p. 233.

67 Fernández de Córdoba, “Michoacán: la historia y sus instrumentos” (5), 1952, pp. 136-137.

68 García Martínez y LiRa González, “Querétaro: la historia y sus instrumentos" (70), 1968, p. 288.

69 Sudo y De los Reyes, “Xalapa: la historia y sus instrumentos”, (96), 1975, p. 611 . 
La meta que originalmente perseguían los artículos de esta serie, de contribuir “a despertar interés por relacionar las actividades de los historiadores del interior con los del centro", ${ }^{70}$ fue difícil de cumplir. En cambio, éstos tuvieron gran utilidad como guías de investigación y abrieron el camino a muchos historiadores interesados en explorar algún tema regional. Muchos más artículos se publicaron en Historia Mexicana, en sus secciones de Testimonios o Examen de Archivos, enfocados a brindar información sobre fuentes documentales en las entidades federativas.

A lo largo del tiempo, cada vez más historiadores se inclinaron hacia la historia subnacional, como refleja la tendencia creciente del número de artículos y reseñas con esta perspectiva publicados en Historia Mexicana (véase la gráfica 1). En total, éstos suman 404 artículos y 282 reseñas sobre libros distintos. Su crecimiento se aceleró a partir de los últimos años de la década de 1960 y a lo largo de los dos siguientes decenios. Durante la década de 1990 su importancia disminuyó, tal vez porque fueron surgiendo revistas especializadas en temas regionales. Sin embargo, la perspectiva subnacional recobró fuerzas en las dos últimas décadas.

Siguiendo la tipología que desarrolló Luis González, dividimos los trabajos entre historia local, historia regional e historia estatal (véase la tabla 1). ${ }^{71}$ Al hacerlo, encontramos que mientras que en la década de 1950 la mayor parte de los trabajos (53.2\%) se referían en sus títulos a un estado o provincia, éstos fueron perdiendo importancia a lo largo del tiempo representando solamente $26.7 \%$ en la década de 2010. Por su parte, los artículos o reseñas cuyos títulos se refieren a una región pasaron de $14.9 \%$ en la década de 1950 a $27.8 \%$ en la de 2010. Los más numerosos son los trabajos que se ocupan de una localidad, representando

70 García Martínez y LiRa GonzÁlez, “Querétaro: la historia y sus instrumentos" (70), 1968, p. 286.

71 La clasificación se realizó con base en sus títulos, si bien cuando existía la duda se exploró su contenido. 


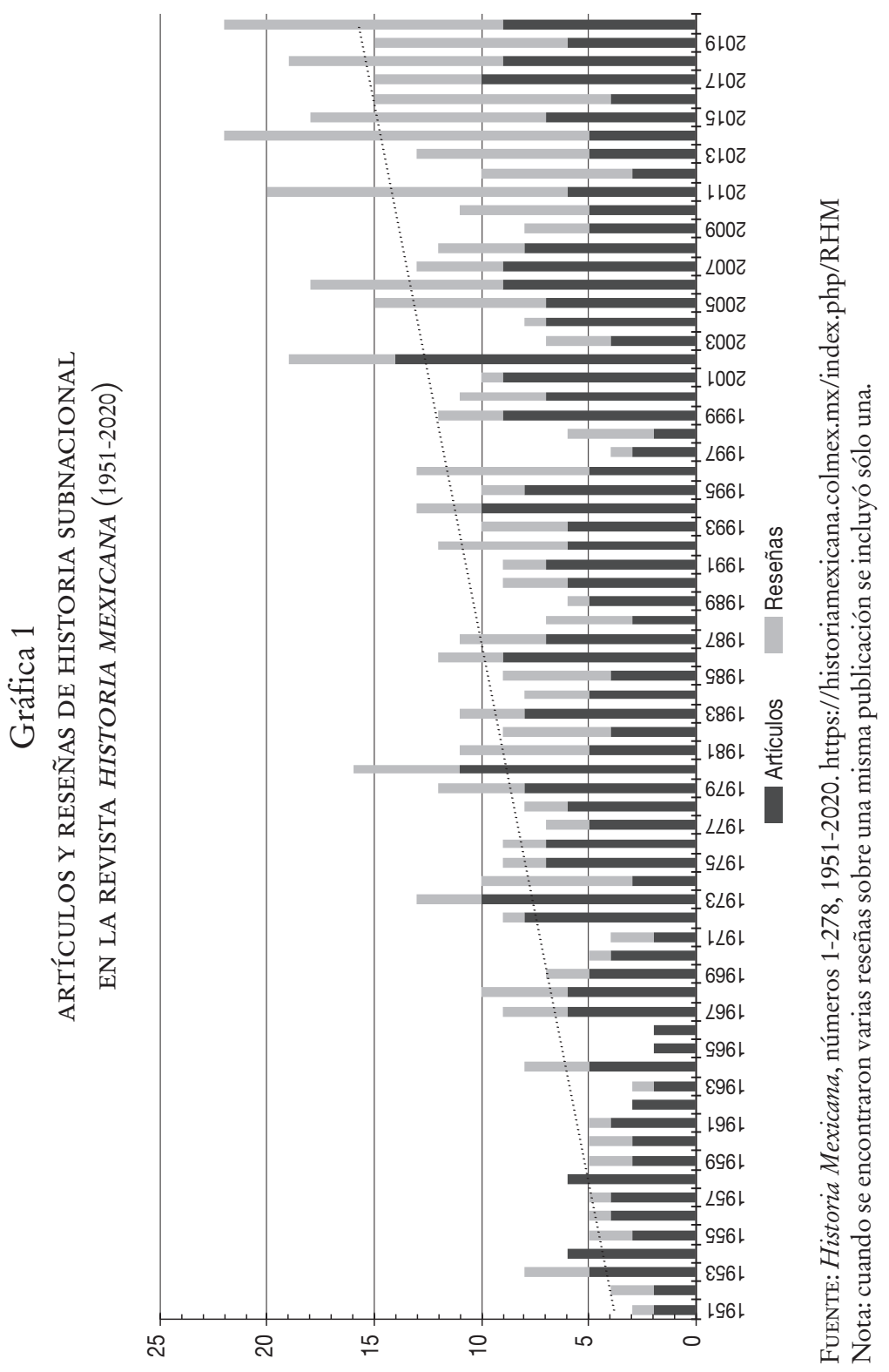


$40.1 \%$ de los trabajos publicados entre 1951 y 2020. El decenio en que su porcentaje fue mayor fue el de 1960 (55.6\%), pero su importancia continuó durante las siguientes décadas, representando en la de 2010 el $45.6 \%$ de los trabajos.

\section{Tabla 1}

ARTÍCULOS Y RESEÑAS EN HISTORIA MEXICANA, SEGÚN EL TIPO DE PERSPECTIVA SUBNACIONAL (1951-2010)

\begin{tabular}{lcccc}
\hline & Regional & Local & Estatal & Total \\
\hline $1950-1959$ & $14.9 \%$ & $31.9 \%$ & $53.2 \%$ & 47 \\
$1960-1969$ & $22.2 \%$ & $55.6 \%$ & $22.2 \%$ & 54 \\
$1970-1979$ & $30.2 \%$ & $40.7 \%$ & $29.1 \%$ & 86 \\
$1980-1989$ & $28.0 \%$ & $32.0 \%$ & $40.0 \%$ & 100 \\
$1990-1999$ & $27.6 \%$ & $33.7 \%$ & $38.8 \%$ & 98 \\
$2000-2009$ & $30.6 \%$ & $39.7 \%$ & $29.8 \%$ & 121 \\
$2010-2020$ & $27.8 \%$ & $45.6 \%$ & $26.7 \%$ & 180 \\
\hline Total & $27.3 \%$ & $40.1 \%$ & $32.7 \%$ & 686 \\
\hline
\end{tabular}

FuENTE: véase la gráfica 1.

La entidad federativa que más atención recibió por parte de los historiadores fue Yucatán, con 36 publicaciones, seguida por Michoacán y Oaxaca con más de 20. A éstas les siguen Puebla, Jalisco, Chiapas y Baja California con 10 o más publicaciones. Varias en cambio tuvieron una sola publicación (Hidalgo, Morelos, Guerrero y Colima) y algunas ninguna (Sinaloa, Nayarit, Campeche). Varios trabajos se refieren a los nombres de los estados durante el periodo virreinal de forma anacrónica. Otros utilizan los nombres de las provincias, de las que la más estudiada fue Nueva Galicia, seguida por Nueva Vizcaya (véase la tabla A1 del apéndice).

En cuanto a las historias locales, gran cantidad de los trabajos se concentra en la ciudad de México, con 99 publicaciones, 
seguida de lejos por Guadalajara y Puebla. También hay varios que estudian pequeños poblados, como Xochimilco, Anenecuilco, Bolaños o Acuecuexco. Asimismo, ocho trabajos estudian distintas haciendas, uno un convento y otro un ingenio (véase la tabla A2 del apéndice).

La forma como los historiadores han identificado en sus títulos a las regiones expresa las distintas perspectivas con las que la disciplina se ha aproximado al concepto de región. El criterio más utilizado y que ha ido en aumento ha sido la dimensión geográfica, ya sea en términos de su localización (Sureste, Noreste), de regiones que son conocidas como tales (Bajío, los Altos), o a partir de un rasgo orográfico o hidrológico (Sierra Gorda, Ciénaga de Chapala) (véase la tabla A3a del apéndice).

Otra forma común de definir una región ha sido considerando el área dominada por un nodo urbano, como hacen Juan Carlos Garavaglia y Juan Carlos Grosso en su artículo sobre indios, campesinos y mercado en la región de Puebla. ${ }^{72}$ Otros trabajos identifican una región a partir de un conjunto de ciudades o poblados, como lo hace Gisela von Wobeser en su artículo sobre el uso del agua en la región de Cuernavaca y Cuautla. ${ }^{73}$ Una ruta alternativa ha sido elegir una zona geográfica de una entidad federativa o provincia o reunir un grupo de estados de la República.

Encontramos también varios trabajos que utilizan una demarcación jurisdiccional civil o religiosa para definir una región, como un obispado, o bien el territorio que ocupaba una explotación económica, como el latifundio de los Sánchez Navarro. Otros trabajos identifican la región como el espacio poblado por determinadas etnias o culturas indígenas. Finalmente, otros han definido las regiones a partir de su característica de frontera,

72 Garavaglia y Grosso, "Indios, campesinos y mercado. La región de Puebla” (182), 1996, pp. 245-278.

73 Wobeser, "El uso del agua en la región de Cuernavaca, Cuautla" (128), 1983, pp. 467-495. 
entendida bien como espacio abierto o como límite administrativo. El primer caso lo ejemplifica el artículo de Woodrow Borah sobre un gobierno provincial de frontera en San Luis Potosí; el segundo, el artículo de Laura Muñoz Mata sobre el Golfo-Caribe en su transición de límite a frontera. ${ }^{74}$

Los artículos y reseñas con una perspectiva subnacional variaron también en términos del periodo estudiado. Como vemos en la tabla 2, los más numerosos han estudiado el periodo virreinal, seguidos por los que se ocupan del siglo XIX (entendido como el periodo que va desde que terminan las guerras de independencia hasta el final del régimen de Porfirio Díaz). Sin embargo, a lo largo del tiempo, la perspectiva subnacional se fue difundiendo hacia todos los periodos históricos.

\section{Tabla 2}

ARTÍCULOS Y RESEÑAS DE HISTORIA SUBNACIONAL EN HISTORIA MEXICANA (1951-2020) POR PERIODO DE ESTUDIO

\begin{tabular}{|c|c|c|c|c|c|c|}
\hline & Virreinal & $\begin{array}{c}\text { Guerra } \\
\text { de Inde- } \\
\text { pendencia }\end{array}$ & $\begin{array}{l}\text { Siglo } \\
X I X\end{array}$ & $\begin{array}{c}\text { Revolu- } \\
\text { ción }\end{array}$ & $\begin{array}{c}\text { Siglo } \\
X X\end{array}$ & General \\
\hline $1950-1959$ & $30.4 \%$ & $4.3 \%$ & $25.5 \%$ & $4.3 \%$ & $0.0 \%$ & $31.9 \%$ \\
\hline 1960-1969 & $38.9 \%$ & $0.0 \%$ & $25.9 \%$ & $5.6 \%$ & $3.7 \%$ & $25.9 \%$ \\
\hline 1970-1979 & $39.5 \%$ & $5.8 \%$ & $21.1 \%$ & $7.0 \%$ & $5.8 \%$ & $19.8 \%$ \\
\hline 1980-1989 & $48.0 \%$ & $0.0 \%$ & $19.0 \%$ & $12.0 \%$ & $12.0 \%$ & $9.0 \%$ \\
\hline 1990-1999 & $40.8 \%$ & $1.0 \%$ & $33.7 \%$ & $9.2 \%$ & $9.2 \%$ & $6.1 \%$ \\
\hline 2000-2009 & $30.6 \%$ & $3.3 \%$ & $31.4 \%$ & $9.9 \%$ & $14.0 \%$ & $10.7 \%$ \\
\hline $2010-2020$ & $35.0 \%$ & $1.7 \%$ & $30.6 \%$ & $7.2 \%$ & $14.4 \%$ & $11.1 \%$ \\
\hline Total & $37.8 \%$ & $2.2 \%$ & $27.7 \%$ & $8.3 \%$ & $10.3 \%$ & $13.7 \%$ \\
\hline
\end{tabular}

FuENTE: véase la gráfica 1.

74 BoraH, "Un gobierno provincial de frontera en San Luis Potosí" (52), 1964, pp. 532-550; MuÑoz Mata, "El Golfo-Caribe, de límite a frontera de México" (226), 2007, pp. 531-563. 
La historia política ha representado alrededor de una cuarta parte de los trabajos subnacionales, porcentaje que se ha mantenido con ligeras variaciones a lo largo del tiempo. Entre 1951 y la década de 1970, también tuvieron gran importancia los trabajos abocados a brindar información sobre las fuentes disponibles en archivos y bibliotecas (véase la tabla 3). Entre las décadas de 1950 y 1970, la historia económica fue ganando relevancia, pasando de $6.4 \%$ a $22.1 \%$ de las publicaciones y manteniéndose en ese nivel durante las siguientes décadas. La historia social es el campo que creció más constantemente, pasando de solo $4.3 \%$ en la década de 1950 a ser el que ocupa el mayor porcentaje de publicaciones en el último decenio, enfocándose muchos de sus trabajos en la ciudad de México. A partir de la década de 1970, se han publicado gran número de artículos que tratan de la historia de la educación en distintas ciudades y pueblos del país. Existen también diversos artículos relacionados con cuestiones geográficas, temas urbanos y más recientemente ambientales, que estudian localidades o regiones particulares, así como varios de carácter general. Un ejemplo destacado de estos últimos es el que realizó Alejandra Moreno Toscano sobre la importancia que tiene para el historiador el examen de la manera como se han denominado en las diversas épocas los accidentes geográficos, las ciudades, los pueblos, etc. ${ }^{75}$ En cuanto a los estudios dedicados a temas demográficos, éstos tuvieron mayor importancia relativa durante las décadas de 1980 y 1990.

75 Moreno Toscano, “Toponimia y análisis histórico” (73), 1969, pp. 1-10. 


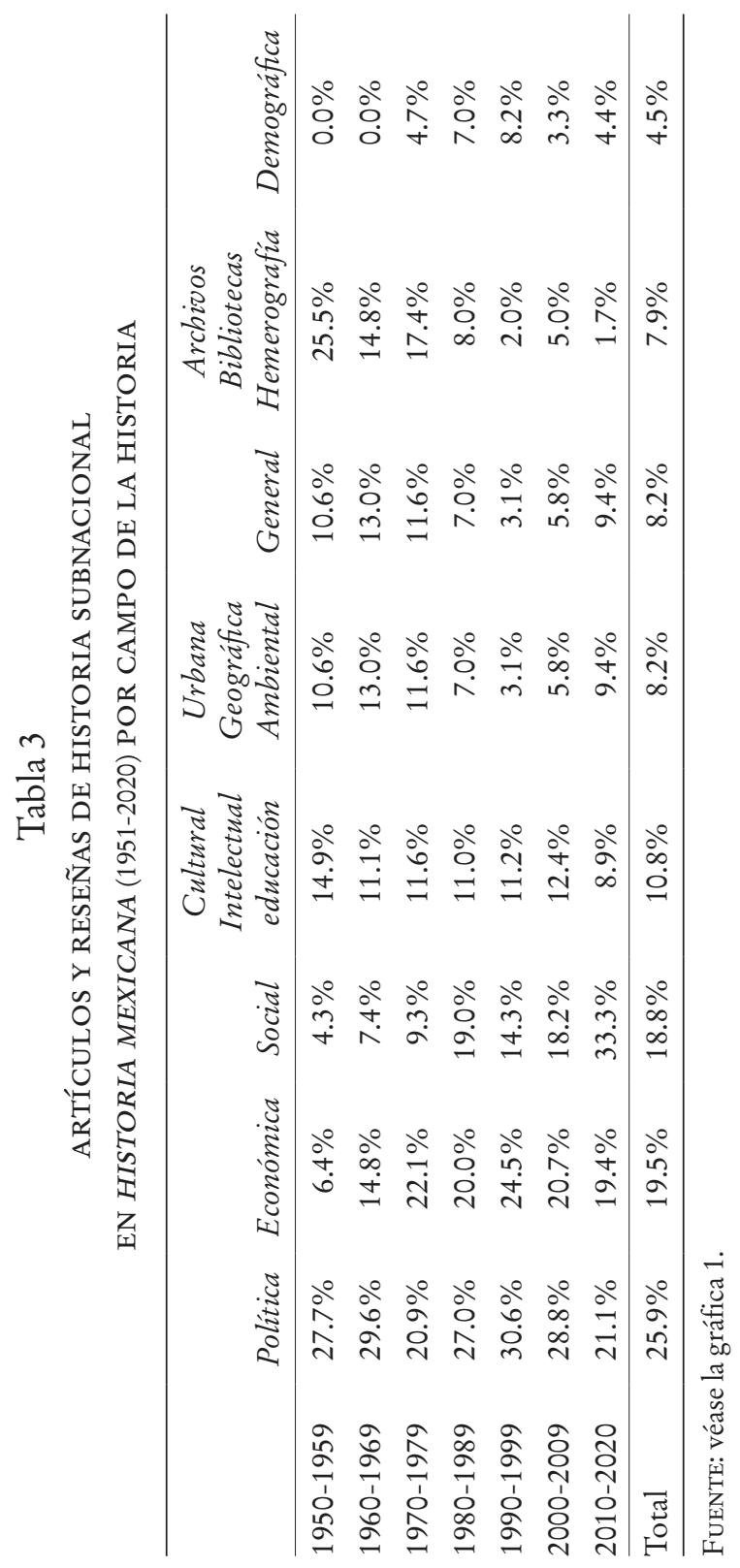




\section{CONCLUSIONES}

A lo largo de estas páginas hemos explorado la evolución del concepto del espacio y su importancia en el quehacer histórico, así como los debates en torno de lo que se entiende por historia regional. Estas discusiones han dejado su huella en las páginas de la revista Historia Mexicana, pues varios de sus interlocutores fueron profesores o egresados del Centro de Estudios Históricos de El Colegio de México.

El análisis de la evolución de la perspectiva subnacional, a la luz de los artículos y reseñas publicados en Historia Mexicana, muestra un sostenido crecimiento a lo largo del tiempo. Al igual que en Europa, en México su auge inició durante la década de 1970. Este estudio muestra también que desde los años cincuenta la revista desempeñó un papel importante en su desarrollo por medio de la publicación de artículos que tenían como objetivo promoverla y aportar herramientas para que los historiadores pudieran acercarse a ella. En los primeros números de la revista podemos observar los enormes retos que enfrentó la disciplina para incursionar en la perspectiva regional y local, y cómo éstos pudieron superarse.

Al paso del tiempo, observamos una creciente importancia de las historias regionales y locales en relación con las dedicadas al estudio histórico de entidades federativas. En cuanto a los periodos históricos a los que se abocaron los trabajos, encontramos que en sus inicios éstos se enfocaron en el periodo virreinal, pero gradualmente las perspectivas subnacionales fueron conquistando todos los periodos. Algo similar resulta en torno al campo de estudio. Mientras que en un inicio la mayor parte de los trabajos se referían a la historia política, con el tiempo éstos fueron abordando también temas de historia económica, social, cultural y otras. En cuanto a los distintos criterios utilizados para definir los espacios de estudio, observamos que a lo largo del tiempo éstos se fueron sofisticando y caracterizando más por 
variables geográficas. Más que las piezas de un mosaico, estas publicaciones se asemejan a las piezas de un caleidoscopio, pues dependiendo del giro que le demos, del objetivo con que nos aproximemos a ellas, se acomodarán de forma distinta para brindarnos una imagen que nos permita comprender mejor algún aspecto de la historia.

El balance general señala que la historia regional y local ha logrado establecerse definitivamente en la historiografía mexicana y ha alcanzado la importancia que vislumbró Luis González y González. Si bien durante la década de 1990 su auge pareciera haber menguado, ésta retomó bríos durante las dos primeras décadas del siglo xxi. Sin embargo, la historia subnacional continúa teniendo tareas pendientes, una de las cuales es ampliar hacia otras ciudades y poblados los estudios que en las últimas décadas se han centrado de forma preponderante en la Ciudad de México.

\section{REFERENCIAS}

Аввотт, Carl, "United States Regional History as an Instructional Field: The Practice of College and University Departments", en Western Historical Quarterly, 21: 2 (1990), pp. 197-217.

Applegate, Celia, "A Europe of Regions: Reflections on the Historiography of Sub-National Places in Modern Times", en The American Historical Review, 104: 4 (1999), pp. 1157-1182.

Arias, Patricia, "Luis González. Microhistoria e historia regional”, en Desacatos, 21 (2006), pp. 177-186.

Cosío Villegas, Daniel (ed.), Historia moderna de México. La República Restaurada. La vida social, México, Hermes, 1956.

García Martínez, Bernardo, "Consideraciones corográficas”, en Cosío Villegas (coord.), Historia general de México, México, El Colegio de México, 1977, vol. I, pp. 5-82.

García Martínez, Bernardo, Las regiones de México. Breviario geográfico e histórico, México, El Colegio de México, 2008. 
González y González, Luis, Pueblo en vilo. Microhistoria de San José de Gracia, México, El Colegio de México, 1968.

González y González, Luis, “Terruño, microhistoria y ciencias sociales”, en Pérez Herrero (comp.), 1991, pp. 23-36.

GonZÁleZ y GonZÁlez, Luis, "Historia regional en sentido riguroso", en Invitación a la microhistoria, México, Clío y El Colegio Nacional, 1997.

González y González, Luis, "Hacia una teoría de la microhistoria”, en Academia Mexicana de la Historia, Discurso de Recepción, 27 de marzo de 1973, pp. 2-12.

GonZÁlez Martínez, Joaquín Roberto, Alvarado y el mundo del agua. Larga duración y conformación geobistórica del Bajo Papaloapan veracruzano, Xalapa, Editora del Gobierno del Estado de Veracruz, 2004.

Heseltine, William B., "The Value of Regional History”, en The Arkansas Historical Quarterly, 7: 1 (1948), pp. 11-19.

Hiernaux, Daniel N. y Alicia Lindon, "El concepto de espacio y el análisis regional”, en Secuencia, 25 (1993), pp. 89-110.

Joseph, Gilbert M., "La nueva historiografía regional de México: una evaluación preliminar”, en Serrano Álvarez (coord.), 1998, pp. 54-62.

Peña, Guillermo de la, "La región: visiones antropológicas”, en SERrano Álvarez (coord.), 1998, pp. 12-24.

PeÑa, Guillermo de la, "Los estudios regionales y la antropología social en México”, en Relaciones, 8 (1981), pp. 43-93.

Pérez Herrero, Pedro (comp.), Región e historia en México (1700-1850). Métodos de análisis regional, México, Instituto de Investigaciones Dr. José María Luis Mora, Universidad Autónoma Metropolitana, 1991.

Reina, Leticia, "Historia regional e historia nacional", en Historias, 29 (oct. 1992-mar. 1993), pp. 131-141.

Serrano Álvarez, Pablo (coord.), Pasado, presente y futuro de la historiografía regional de México: memorias del Congreso de Historiografía regional, México, Universidad Nacional Autónoma de México, 1998, vol. 1. 
VAN Young, Eric, "Haciendo historia regional. Consideraciones metodológicas y teóricas”, en Anuario IEHS, 2 (1987), pp. 255-281.

VILAR, Pierre, "Sobre los fundamentos de las estructuras nacionales", en Historias, 16 (1978), pp. 5-16.

Viqueira, Juan Pedro, "Regiones naturales, regiones nominales y regiones vividas", en Sotavento, Revista de Historia, Sociedad y Cultura, 3 (invierno 1997-1998), pp. 107-117.

Weber, Eugene, My France: Politics, Culture, Myth, Cambridge, Mass., Belknap Press of Harvard University Press, 1991.

Withers, Charles W. J., "Place and the 'Spatial Turn' in Geography and in History", en Journal of the History of Ideas, 70: 4 (2009), pp. 637-658. 


\title{
Apéndice
}

\author{
Tabla A1
}

ARTÍCULOS Y RESEÑAS EN HISTORIA MEXICANA (1951-2020) POR ENTIDAD FEDERATIVA

\begin{tabular}{|c|c|c|c|c|c|c|c|c|}
\hline & $\begin{array}{c}1950- \\
1959\end{array}$ & $\begin{array}{r}1960 \\
1969\end{array}$ & $\begin{array}{c}1970- \\
1979\end{array}$ & $\begin{array}{r}1980- \\
1989\end{array}$ & $\begin{array}{c}1990- \\
1999\end{array}$ & $\begin{array}{c}2000 \\
2009\end{array}$ & $\begin{array}{r}2010- \\
2020\end{array}$ & Total \\
\hline Yucatán & 2 & 2 & 3 & 7 & 9 & 3 & 10 & 36 \\
\hline Michoacán & 2 & & 1 & 1 & 5 & 7 & 8 & 24 \\
\hline Oaxaca & 4 & & 3 & 3 & 2 & 4 & 5 & 21 \\
\hline Puebla & & 2 & 3 & 2 & 4 & 1 & 2 & 14 \\
\hline Jalisco & 2 & & 5 & 2 & 1 & 2 & 1 & 13 \\
\hline Chiapas & & 1 & 2 & 1 & 2 & 1 & 3 & 10 \\
\hline Baja California & 3 & 1 & & 1 & & 3 & 1 & 9 \\
\hline Veracruz & 1 & & 2 & 2 & 1 & 2 & 1 & 9 \\
\hline Chihuahua & 1 & 1 & 2 & 1 & & 3 & 1 & 9 \\
\hline Tlaxcala & 1 & & 2 & 2 & 1 & 1 & 2 & 9 \\
\hline \multicolumn{9}{|l|}{ Alta California/ } \\
\hline California & 2 & 1 & 1 & 1 & 3 & 1 & & 9 \\
\hline Sonora & & 1 & & 1 & 1 & 2 & 4 & 9 \\
\hline \multicolumn{9}{|l|}{ Estado de } \\
\hline México & & & 2 & 2 & 3 & 1 & 1 & 9 \\
\hline Nueva Galicia & 3 & & 2 & 1 & 1 & & & 7 \\
\hline San Luis Potosí & & 1 & 1 & 2 & 1 & 1 & 1 & 7 \\
\hline Texas & 1 & & & 1 & 4 & & & 6 \\
\hline \multicolumn{9}{|l|}{ Nuevo Reino } \\
\hline de León & 1 & & & 1 & & 1 & 1 & 4 \\
\hline Zacatecas & 1 & & & 1 & 1 & 1 & & 4 \\
\hline Querétaro & & 2 & & 1 & & & 1 & 4 \\
\hline Aguascalientes & & & & 1 & 1 & 1 & 1 & 4 \\
\hline Durango & & 1 & 1 & 1 & & & & 3 \\
\hline Nueva Vizcaya & & & & 1 & & 1 & 1 & 3 \\
\hline Coahuila & & & & 1 & & 1 & 1 & 3 \\
\hline Distrito Federal & & & & & 2 & & 1 & 3 \\
\hline Tabasco & & & & & & & 3 & 3 \\
\hline Nuevo México & & & 1 & 1 & & & & 2 \\
\hline Tamaulipas & & & & 1 & & & 1 & 2 \\
\hline Guanajuato & & & & & 1 & & 1 & 2 \\
\hline Quintana Roo & & & & & 1 & & 1 & 2 \\
\hline
\end{tabular}




\section{Tabla A1}

ARTÍCULOS Y RESEÑAS EN HISTORIA MEXICANA (1951-2020) POR ENTIDAD FEDERATIVA (concluye)

\begin{tabular}{lcccccccc}
\hline & $1950-$ & $1960-$ & $1970-$ & $1980-$ & $1990-$ & $2000-$ & 2010 & \\
& 1959 & 1969 & 1979 & 1989 & 1999 & 2009 & 2020 & Total \\
\hline $\begin{array}{l}\text { Nuevo León } \\
\text { Nuevo }\end{array}$ & 1 & & & & & & & 1 \\
$\quad$ Santander & & & 1 & & & & & 1 \\
$\begin{array}{l}\text { Hidalgo } \\
\text { Morelos }\end{array}$ & & & 1 & & & & & 1 \\
$\begin{array}{l}\text { Guerrero } \\
\text { Colima }\end{array}$ & & & & 1 & & & & 1 \\
$\begin{array}{l}\text { Total entidades } \\
\quad \text { federativas }\end{array}$ & 25 & 13 & 33 & 41 & 44 & 38 & 52 & 246 \\
$\begin{array}{l}\text { Pub. Generales } \\
\quad \text { de Hist. Sub. }\end{array}$ & 2 & 5 & 10 & 3 & 4 & 10 & 20 & 54 \\
\hline
\end{tabular}

FuENTE: véase la gráfica 1. 


\section{Tabla A2}

ARTÍCULOS Y RESEÑAS EN HISTORIA MEXICANA (1951-2020)

POR LOCALIDAD

\begin{tabular}{|c|c|c|c|c|c|c|c|c|}
\hline & $\begin{array}{c}1950- \\
1959\end{array}$ & $\begin{array}{c}1960- \\
1969\end{array}$ & $\begin{array}{r}1970- \\
1979\end{array}$ & $\begin{array}{c}1980- \\
1989\end{array}$ & $\begin{array}{r}1990- \\
1999\end{array}$ & $\begin{array}{c}2000 \\
2009\end{array}$ & $\begin{array}{c}2010- \\
2020\end{array}$ & Total \\
\hline \multicolumn{9}{|l|}{ Ciudad de } \\
\hline México & 2 & 4 & 8 & 12 & 7 & 15 & 51 & 99 \\
\hline Guadalajara & 2 & 3 & & & & 2 & 3 & 10 \\
\hline Puebla & & 1 & 1 & 3 & 1 & 2 & 1 & 9 \\
\hline Veracruz & & 2 & & 2 & 1 & 1 & & 6 \\
\hline Monterrey & & 1 & & & & 2 & 1 & 4 \\
\hline Xalapa & & & 3 & & & 1 & & 4 \\
\hline Zacatecas & & & 1 & & 2 & 1 & & 4 \\
\hline Bolaños & & 1 & 1 & & & & 1 & 3 \\
\hline Toluca & & & 1 & & & 2 & & 3 \\
\hline Xochimilco & & & & 1 & & 2 & & 3 \\
\hline San Luis Potosí & & & & 1 & & & 2 & 3 \\
\hline Anenecuilco & 1 & & & & 1 & & & 2 \\
\hline Cananea & 1 & 1 & & & & & & 2 \\
\hline Mérida & & 1 & & & & 1 & & 2 \\
\hline Atlixco & & 1 & & & & 1 & & 2 \\
\hline Orizaba & & 1 & & & & & 1 & 2 \\
\hline Zamora & & & & 1 & 1 & & & 2 \\
\hline \multicolumn{9}{|l|}{ San Luis de la } \\
\hline Paz, Gto. & & & & 1 & 1 & & & 2 \\
\hline Valladolid, Mich. & & & & & 1 & 1 & & 2 \\
\hline Papantla, Ver. & & & & & & & 2 & 2 \\
\hline Acuecuexco & 1 & & & & & & & 1 \\
\hline Mochis & 1 & & & & & & & 1 \\
\hline Colima & 1 & & & & & & & 1 \\
\hline Tlaltenango & 1 & & & & & & & 1 \\
\hline Tampico & 1 & & & & & & & 1 \\
\hline Tacubaya & & 1 & & & & & & 1 \\
\hline Cuernavaca & & 1 & & & & & & 1 \\
\hline Durango & & 1 & & & & & & 1 \\
\hline \multicolumn{9}{|l|}{ Salinas de Peñón } \\
\hline Blanco & & 1 & & & & & & 1 \\
\hline Tlaxcala & & 1 & & & & & & 1 \\
\hline \multicolumn{9}{|l|}{ San Juan } \\
\hline Hueyapan & & 1 & & & & & & 1 \\
\hline
\end{tabular}




\section{Tabla A2}

ARTÍCULOS Y RESEÑAS EN HISTORIA MEXICANA (1951-2020) POR LOCALIDAD (continúa)

\begin{tabular}{|c|c|c|c|c|c|c|c|c|}
\hline & $\begin{array}{c}1950- \\
1959\end{array}$ & $\begin{array}{c}1960- \\
1969\end{array}$ & $\begin{array}{c}1970- \\
1979\end{array}$ & $\begin{array}{c}1980- \\
1989\end{array}$ & $\begin{array}{c}1990- \\
1999\end{array}$ & $\begin{array}{r}2000- \\
2009\end{array}$ & $\begin{array}{r}2010 \\
2020\end{array}$ & Total \\
\hline Acámbaro & & 1 & & & & & & 1 \\
\hline Cuauhtinchán & & 1 & & & & & & 1 \\
\hline Zinacantán & & 1 & & & & & & 1 \\
\hline Oaxaca & & 1 & & & & & & 1 \\
\hline \multicolumn{9}{|l|}{ San José de } \\
\hline Gracia & & 1 & & & & & & 1 \\
\hline Zongolica & & & 1 & & & & & 1 \\
\hline Guanajuato & & & 1 & & & & & 1 \\
\hline Cholula & & & 1 & & & & & 1 \\
\hline Polotitlán & & & 1 & & & & & 1 \\
\hline \multicolumn{9}{|l|}{ Dolores, } \\
\hline Hidalgo & & & 1 & & & & & 1 \\
\hline \multicolumn{9}{|l|}{ Santiestaban } \\
\hline del Puerto & & & 1 & & & & & 1 \\
\hline Tula & & & & 1 & & & & 1 \\
\hline Ocoyoacac & & & & 1 & & & & 1 \\
\hline \multicolumn{9}{|l|}{ Pueblos de Santa } \\
\hline $\mathrm{Fe}$ & & & & 1 & & & & 1 \\
\hline \multicolumn{9}{|l|}{ Pisaflores, } \\
\hline Hidalgo & & & & 1 & & & & 1 \\
\hline \multicolumn{9}{|l|}{ Santa Cruz } \\
\hline de Tlatelolco & & & & 1 & & & & 1 \\
\hline Ixtapan de la Sal & & & & 1 & & & & 1 \\
\hline Misantla & & & & 1 & & & & 1 \\
\hline Parral & & & & & 1 & & & 1 \\
\hline \multicolumn{9}{|l|}{ Salinas de } \\
\hline Tepopoxtla & & & & & 1 & & & 1 \\
\hline Córdoba & & & & & 1 & & & 1 \\
\hline Real del Monte & & & & & 1 & & & 1 \\
\hline Chalco & & & & & 1 & & & 1 \\
\hline Tepotzotlán & & & & & 1 & & & 1 \\
\hline \multicolumn{9}{|l|}{ San Juan de los } \\
\hline Lagos & & & & & 1 & & & 1 \\
\hline Amecameca & & & & & 1 & & & 1 \\
\hline Chihuahua & & & & & & 1 & & 1 \\
\hline
\end{tabular}




\section{Tabla A2}

ARTÍCULOS Y RESEÑAS EN HISTORIA MEXICANA (1951-2020)

POR LOCALIDAD (continúa)

\begin{tabular}{|c|c|c|c|c|c|c|c|c|}
\hline & $\begin{array}{l}1950- \\
1959\end{array}$ & $\begin{array}{r}1960- \\
1969\end{array}$ & $\begin{array}{r}1970- \\
1979\end{array}$ & $\begin{array}{r}1980- \\
1989\end{array}$ & $\begin{array}{l}1990- \\
1999\end{array}$ & $\begin{array}{l}2000- \\
2009\end{array}$ & $\begin{array}{l}2010- \\
2020\end{array}$ & Total \\
\hline \multicolumn{9}{|l|}{ Coalcomán, } \\
\hline Mich. & & & & & & 1 & & 1 \\
\hline Tlajomulco & & & & & & 1 & & 1 \\
\hline Necaxa & & & & & & 1 & & 1 \\
\hline \multicolumn{9}{|l|}{ Santo Domingo } \\
\hline \multicolumn{9}{|l|}{ Tepenene, } \\
\hline Oax. & & & & & & 1 & & 1 \\
\hline Jerez & & & & & & 1 & & 1 \\
\hline Querétaro & & & & & & 1 & & 1 \\
\hline Tocumbo, Mich. & & & & & & 1 & & 1 \\
\hline Ixtlán, Oax & & & & & & 1 & & 1 \\
\hline Tehuacán & & & & & & 1 & & 1 \\
\hline Tepexi de la Seda & & & & & & & 1 & 1 \\
\hline Tucson, Arizona & & & & & & & 1 & 1 \\
\hline \multicolumn{9}{|l|}{ Ixtlahuaca, Edo. } \\
\hline Méx. & & & & & & & 1 & 1 \\
\hline \multicolumn{9}{|l|}{ Villa Alta, } \\
\hline Oaxaca & & & & & & & 1 & 1 \\
\hline Iztapalapa & & & & & & & 1 & 1 \\
\hline Coapa & & & & & & & 1 & 1 \\
\hline Mazapil & & & & & & & 1 & 1 \\
\hline \multicolumn{9}{|l|}{ San Cristóbal de } \\
\hline Las Casas & & & & & & & 1 & 1 \\
\hline Tijuana & & & & & & & 1 & 1 \\
\hline \multicolumn{9}{|l|}{ San Juan } \\
\hline \multicolumn{9}{|l|}{ Teotihuacán, } \\
\hline Edo. Méx. & & & & & & & 1 & 1 \\
\hline Cuetzala & & & & & & & 1 & 1 \\
\hline Tultotepec & & & & & & & 1 & 1 \\
\hline Islas Marías & & & & & & & 1 & 1 \\
\hline Aguascalientes & & & & & & & 1 & 1 \\
\hline \multicolumn{9}{|l|}{ Hacienda de } \\
\hline $\begin{array}{l}\text { Santa Lucía, } \\
\text { meseta central }\end{array}$ & & & 1 & & & & & 1 \\
\hline
\end{tabular}


Tabla A2

ARTÍCULOS Y RESEÑAS EN HISTORIA MEXICANA (1951-2020) POR LOCALIDAD (concluye)

\begin{tabular}{|c|c|c|c|c|c|c|c|c|}
\hline & $\begin{array}{c}1950- \\
1959\end{array}$ & $\begin{array}{c}1960- \\
1969\end{array}$ & $\begin{array}{c}1970- \\
1979\end{array}$ & $\begin{array}{c}1980- \\
1989\end{array}$ & $\begin{array}{c}1990- \\
1999\end{array}$ & $\begin{array}{c}2000- \\
2009\end{array}$ & $\begin{array}{l}2010- \\
2020\end{array}$ & Total \\
\hline $\begin{array}{l}\text { Hacienda de San } \\
\text { Pedro Jorullo, } \\
\text { Michoacán }\end{array}$ & & & 1 & & & & & 1 \\
\hline $\begin{array}{l}\text { Hacienda de San } \\
\text { Bartolomé }\end{array}$ & & & & & & & & \\
\hline Tepetates & & & 1 & & & & & 1 \\
\hline $\begin{array}{l}\text { Haciendas de } \\
\text { Mazaquiahuac }\end{array}$ & & & & & & & & \\
\hline $\begin{array}{l}\text { y El Rosario } \\
\text { Hacienda de }\end{array}$ & & & & 1 & & & & 1 \\
\hline Santa Ana & & & & & & & & \\
\hline Aragón & & & & 1 & & & & 1 \\
\hline $\begin{array}{l}\text { Hacienda de } \\
\text { "La Concha", }\end{array}$ & & & & & & & & \\
\hline La Laguna & & & & 1 & & & & 1 \\
\hline Hacienda de los & & & & & & & & \\
\hline $\begin{array}{l}\text { Santiago en } \\
\text { Tecali, Puebla }\end{array}$ & & & & & 1 & & & 1 \\
\hline Hacienda de & & & & & & & & \\
\hline Bocas, SLP & & & & & 1 & & & 1 \\
\hline $\begin{array}{l}\text { Convento de } \\
\text { Santa Clara } \\
\text { de Querétaro }\end{array}$ & & & 1 & & & & & 1 \\
\hline $\begin{array}{l}\text { Ingenio } \\
\text { "El Modelo", }\end{array}$ & & & & & & & & \\
\hline Veracruz & & & & 1 & & & & 1 \\
\hline Total & 11 & 27 & 25 & 32 & 25 & 41 & 76 & 237 \\
\hline
\end{tabular}

FuENTE: véase la gráfica 1. 


\section{Tabla A3a}

ARTÍCULOS Y RESEÑAS EN HISTORIA MEXICANA (1951-2020). POR CRITERIOS DE DELIMITACIÓN DE REGIONES:

VARIABLES GEOGRÁFICAS

\begin{tabular}{|c|c|c|c|c|c|c|c|c|}
\hline & $\begin{array}{l}1950- \\
1959\end{array}$ & $\begin{array}{c}1960- \\
1969\end{array}$ & $\begin{array}{c}1970- \\
1979\end{array}$ & $\begin{array}{c}1980- \\
1989\end{array}$ & $\begin{array}{c}1990- \\
1999\end{array}$ & $\begin{array}{l}2000- \\
2009\end{array}$ & $\begin{array}{l}2010- \\
2020\end{array}$ & Total \\
\hline \multicolumn{9}{|l|}{ LOCALIZACIÓN } \\
\hline Sureste & & 1 & & & 1 & & & 2 \\
\hline \multicolumn{9}{|l|}{$\begin{array}{l}\text { México central/ } \\
\text { centro de }\end{array}$} \\
\hline México & & 1 & & 2 & 2 & 3 & & 8 \\
\hline Noreste & & 1 & & & 1 & & 1 & 3 \\
\hline Sur & & & 1 & & & 1 & & 2 \\
\hline Noroeste & & & & 1 & & 2 & & 3 \\
\hline Norte & & & & & & 2 & 4 & 6 \\
\hline Costa oeste & & & & & 1 & & & 1 \\
\hline Sur-Sureste & & & & & 1 & & & 1 \\
\hline Golfo de México & & & & & & 1 & & 1 \\
\hline Golfo-Caribe & & & & & & 1 & & 1 \\
\hline \multicolumn{9}{|l|}{ Tierras bajas } \\
\hline mexicanas & & & & 1 & & & & 1 \\
\hline Sur y centro & & & & & & & 1 & 1 \\
\hline Centro-norte & & & & & & & 1 & 1 \\
\hline Total & 0 & 3 & 1 & 4 & 6 & 10 & 7 & 31 \\
\hline \multicolumn{9}{|c|}{ REGIONES GEOGRÁFICAS } \\
\hline \multicolumn{9}{|l|}{ Istmo de } \\
\hline Tehuantepec & 1 & & & & & 1 & 2 & 4 \\
\hline Bajío & & & 1 & & & & 2 & 3 \\
\hline \multicolumn{9}{|l|}{ Provincias } \\
\hline Internas & & & 1 & & & & 2 & 3 \\
\hline \multicolumn{9}{|l|}{ Provincias } \\
\hline $\begin{array}{l}\text { Internas } \\
\text { de Oriente }\end{array}$ & & & 1 & & & & & 1 \\
\hline Los Altos & & & & 1 & & & & 1 \\
\hline \multicolumn{9}{|l|}{ La Laguna/ } \\
\hline Comarca & & & & & & & & \\
\hline Lagunera & & & & 1 & 2 & 2 & & 5 \\
\hline Tierra caliente & & & & & 1 & & & 1 \\
\hline Petén & & & & & & 1 & & 1 \\
\hline
\end{tabular}


Tabla A3a

ARTÍCULOS Y RESEÑAS EN HISTORIA MEXICANA (1951-2020). POR CRITERIOS DE DELIMITACIÓN DE REGIONES: VARIABLES GEOGRÁFICAS (concluye)

\begin{tabular}{|c|c|c|c|c|c|c|c|c|}
\hline & $\begin{array}{c}1950 \\
1959\end{array}$ & $\begin{array}{c}1960 \\
1969\end{array}$ & $\begin{array}{r}1970 \\
1979\end{array}$ & $\begin{array}{c}1980- \\
1989\end{array}$ & $\begin{array}{c}1990- \\
1999\end{array}$ & $\begin{array}{r}2000 \\
2009\end{array}$ & $\begin{array}{c}2010 \\
2020\end{array}$ & Total \\
\hline \multicolumn{9}{|l|}{ Llas Huastecas/ } \\
\hline la Huasteca & & & & & & 2 & 1 & 3 \\
\hline Huasteca serrana & & & & & & & 1 & 1 \\
\hline Total & 1 & 0 & 3 & 2 & 3 & 6 & 8 & 23 \\
\hline \multicolumn{9}{|c|}{ OROGRAFÍA O HIDROGRAFÍA } \\
\hline Mixteca & & & 1 & & & & & 1 \\
\hline Mixteca Alta & & 1 & & 1 & & & & 2 \\
\hline Mixteca Costera & & & & 1 & & & & 1 \\
\hline la Tarahumara & & & & 2 & & & & 2 \\
\hline Sierra Gorda & & & 1 & & & & & 1 \\
\hline \multicolumn{9}{|l|}{ Sierra Norte } \\
\hline de Puebla & & & & & & 1 & & 1 \\
\hline Sierra zapoteca & & & & & & & 1 & 1 \\
\hline \multicolumn{9}{|l|}{$\begin{array}{c}\text { Sierra oriental } \\
\text { del valle de }\end{array}$} \\
\hline Toluca & & & & & & & 1 & 1 \\
\hline Sierra de Sonora & & & & & & & 1 & 1 \\
\hline Sierra del Nayar & & & & & & & 1 & 1 \\
\hline \multicolumn{9}{|l|}{ Meseta } \\
\hline purépecha & & & & & & & 1 & 1 \\
\hline \multicolumn{9}{|l|}{ Ciénaga de } \\
\hline Chapala & & & & & 1 & & & 1 \\
\hline \multicolumn{9}{|l|}{ Pueblos del Río } \\
\hline Blanco & & & & & & 1 & & 1 \\
\hline \multicolumn{9}{|l|}{ Cuenca del río } \\
\hline Yaqui & & & & & & & 1 & 1 \\
\hline Valle del Yaqui & & & & & & 1 & & 1 \\
\hline Total & 0 & 1 & 2 & 4 & 1 & 3 & 6 & 17 \\
\hline \multicolumn{9}{|l|}{ TOTAL VARIABLES } \\
\hline GEOGRÁFICAS & 1 & 4 & 6 & 10 & 10 & 19 & 21 & 71 \\
\hline
\end{tabular}

FuENTE: véase la gráfica 1. 
Tabla A3b

ARTÍCULOS Y RESEÑAS EN HISTORIA MEXICANA (1951-2020).

REGIONES POR CRITERIOS DE DELIMITACIÓN DE REGIONES:

EN TORNO A LOCALIDADES O ENTIDADES FEDERATIVAS

\begin{tabular}{|c|c|c|c|c|c|c|c|c|}
\hline & $\begin{array}{c}1950- \\
1959\end{array}$ & $\begin{array}{c}1960- \\
1969\end{array}$ & $\begin{array}{r}1970 \\
1979\end{array}$ & $\begin{array}{c}1980- \\
1989\end{array}$ & $\begin{array}{c}1990- \\
1999\end{array}$ & $\begin{array}{c}2000- \\
2009\end{array}$ & $\begin{array}{c}2010- \\
2020\end{array}$ & Total \\
\hline \multicolumn{9}{|c|}{ REGIONES EN TORNO A CIUDADES O POBLADOS } \\
\hline Valle de Oaxaca & & & 2 & & 1 & & & 3 \\
\hline \multicolumn{9}{|l|}{ Valle de } \\
\hline Xochicalco & & & 1 & & & & & 1 \\
\hline Valle de México & & & & 1 & & & 1 & 2 \\
\hline Valle de Orizaba & & & & & & & 1 & 1 \\
\hline \multicolumn{9}{|l|}{ Guadalajara y su } \\
\hline región & & & 1 & & 1 & & & 2 \\
\hline \multicolumn{9}{|l|}{ Región de Tlapa, } \\
\hline Guerrero & & & & 1 & & 1 & & 2 \\
\hline \multicolumn{8}{|l|}{ Monterrey y } & \\
\hline regional & & & & & 1 & & & 1 \\
\hline \multicolumn{9}{|l|}{$\begin{array}{c}\text { Tepeaca y su } \\
\text { entorno }\end{array}$} \\
\hline agrario & & & & & 1 & & & 1 \\
\hline \multicolumn{9}{|l|}{ Acolhuacán } \\
\hline septentrional & & & 1 & & & & & 1 \\
\hline Bajío zamorano & & & & & 1 & & & 1 \\
\hline \multicolumn{9}{|l|}{ Suroeste de la } \\
\hline \multicolumn{9}{|l|}{ Ciudad de } \\
\hline México & & & & & & & 1 & 1 \\
\hline \multicolumn{9}{|l|}{ Departamento } \\
\hline de Acayucan & & & 1 & & & & & 1 \\
\hline \multicolumn{9}{|l|}{ Distrito de } \\
\hline Cuernavaca & & & & & & 1 & & 1 \\
\hline Total & 0 & 0 & 6 & 2 & 5 & 2 & 3 & 18 \\
\hline \multicolumn{8}{|c|}{ GRUPOS DE CIUDADES O POBLADOS } & \\
\hline y Quecholac & & & & 1 & & & & 1 \\
\hline \multicolumn{9}{|l|}{ Tepeapulco y } \\
\hline Tulancingo & & & & 1 & & & & 1 \\
\hline
\end{tabular}




\section{Tabla A3b}

ARTÍCULOS Y RESEÑAS EN HISTORIA MEXICANA (1951-2020). REGIONES POR CRITERIOS DE DELIMITACIÓN DE REGIONES: EN TORNO A LOCALIDADES O ENTIDADES FEDERATIVAS (continúa)

\begin{tabular}{|c|c|c|c|c|c|c|c|c|}
\hline & $\begin{array}{c}1950- \\
1959\end{array}$ & $\begin{array}{c}1960- \\
1969\end{array}$ & $\begin{array}{r}1970- \\
1979\end{array}$ & $\begin{array}{c}1980- \\
1989\end{array}$ & $\begin{array}{c}1990- \\
1999\end{array}$ & $\begin{array}{l}2000 \\
2009\end{array}$ & $\begin{array}{l}2010 \\
2020\end{array}$ & Total \\
\hline \multicolumn{9}{|l|}{ Real del Monte } \\
\hline y Pachuca & & & & & 1 & & & 1 \\
\hline \multicolumn{9}{|l|}{ Sultepec y } \\
\hline El Oro & & & & & & 1 & & 1 \\
\hline \multicolumn{9}{|l|}{ Guadalajara y } \\
\hline Valladolid & & & & & & 1 & & 1 \\
\hline \multicolumn{9}{|l|}{ Región de } \\
\hline \multicolumn{9}{|l|}{ Cueranavaca } \\
\hline \multicolumn{9}{|l|}{ y Cuautla } \\
\hline Amilpas & & & & 1 & & 1 & & 2 \\
\hline \multicolumn{9}{|l|}{ Totatiche y } \\
\hline \multicolumn{9}{|l|}{ Cañón de } \\
\hline Bolaños & & & & & & & 1 & 1 \\
\hline \multicolumn{9}{|l|}{ Franja costera: } \\
\hline \multicolumn{9}{|l|}{ Culiacán y } \\
\hline Chiametla & & & & & & & 1 & 1 \\
\hline Total & 0 & 0 & 0 & 3 & 1 & 3 & 2 & 9 \\
\hline \multicolumn{9}{|l|}{ TOTAL CIUDADES } \\
\hline O POBLADOS & 0 & 0 & 6 & 5 & 6 & 5 & 5 & 27 \\
\hline \multicolumn{9}{|c|}{$\begin{array}{l}\text { REGIONES QUE FORMAN PARTE DE ENTIDADES FEDERATIVAS O PROVINCIAS } \\
\text { Nueva Vizcaya }\end{array}$} \\
\hline occidental & & 1 & & & & & & 1 \\
\hline \multicolumn{9}{|l|}{ Parte meridional } \\
\hline de Puebla & & & 1 & & & & & 1 \\
\hline Sur de Veracruz & & & 1 & & & 1 & & 2 \\
\hline Altos de Chiapas & & & & 1 & & & & 1 \\
\hline \multicolumn{9}{|l|}{ Oriente de San } \\
\hline \multicolumn{9}{|l|}{ Luis Potosí/ } \\
\hline \multicolumn{9}{|l|}{ Oriente } \\
\hline Potosino & & & & 1 & & & 1 & 2 \\
\hline
\end{tabular}


Tabla A3b

ARTÍCULOS Y RESEÑAS EN HISTORIA MEXICANA (1951-2020). REGIONES POR CRITERIOS DE DELIMITACIÓN DE REGIONES: EN TORNO A LOCALIDADES O ENTIDADES FEDERATIVAS (concluye)

\begin{tabular}{|c|c|c|c|c|c|c|c|c|}
\hline & $\begin{array}{c}1950- \\
1959\end{array}$ & $\begin{array}{c}1960- \\
1969\end{array}$ & $\begin{array}{c}1970- \\
1979\end{array}$ & $\begin{array}{r}1980 \\
1989\end{array}$ & $\begin{array}{c}1990- \\
1999\end{array}$ & $\begin{array}{r}2000 \\
2009\end{array}$ & $\begin{array}{c}2010- \\
2020\end{array}$ & Total \\
\hline \multicolumn{9}{|l|}{ Valles centrales } \\
\hline de Oaxaca & & & & & & 1 & & 1 \\
\hline \multicolumn{9}{|l|}{ Oriente } \\
\hline de Durango & & & & & & 1 & & 1 \\
\hline Total & 0 & 1 & 2 & 2 & 0 & 3 & 1 & 9 \\
\hline \multicolumn{9}{|c|}{ GRUPOS DE ENTIDADES FEDERATIVAS } \\
\hline \multicolumn{9}{|l|}{ Chiapas y } \\
\hline Tabasco & & & & 1 & & & & 1 \\
\hline \multicolumn{9}{|l|}{ Zacatecas y San } \\
\hline Luis Potosí & & & & 1 & & & & 1 \\
\hline las Californias & & & & 1 & & 1 & & 2 \\
\hline \multicolumn{9}{|l|}{ Región Puebla- } \\
\hline Tlaxcala & & & & 1 & 2 & & & 3 \\
\hline \multicolumn{9}{|l|}{ Sinaloa y Baja } \\
\hline California & & & & & & 1 & & 1 \\
\hline \multicolumn{9}{|l|}{ Oaxaca y } \\
\hline Yucatán & & & & & & & 1 & 1 \\
\hline \multicolumn{9}{|l|}{ Nuevo León } \\
\hline y Coahuila & & & & & & & 1 & 1 \\
\hline Total & 0 & 0 & 0 & 4 & 2 & 2 & 2 & 10 \\
\hline \multicolumn{9}{|l|}{ TOTAL POR } \\
\hline \multicolumn{9}{|l|}{ ENTIDADES } \\
\hline \multicolumn{9}{|l|}{ FEDERATIVAS } \\
\hline O PROVINCIAS & 0 & 1 & 2 & 6 & 2 & 5 & 3 & 19 \\
\hline
\end{tabular}

FUENTE: véase la gráfica 1. 


\section{Tabla A3c}

ARTÍCULOS Y RESEÑAS EN HISTORIA MEXICANA (1951-2020). REGIONES POR CRITERIOS DE DELIMITACIÓN DE REGIONES: OTROS CRITERIOS

\begin{tabular}{|c|c|c|c|c|c|c|c|c|}
\hline & $\begin{array}{c}1950- \\
1959\end{array}$ & $\begin{array}{c}1960- \\
1969\end{array}$ & $\begin{array}{c}1970- \\
1979\end{array}$ & $\begin{array}{c}1980- \\
1989\end{array}$ & $\begin{array}{c}1990- \\
1999\end{array}$ & $\begin{array}{c}2000- \\
2009\end{array}$ & $\begin{array}{l}2010 \\
2020\end{array}$ & Total \\
\hline \multicolumn{9}{|c|}{$\begin{array}{l}\text { EN TORNO A ORGANIZACIONES ECONOMICAS, CIVILES O RELIGIOSAS } \\
\text { Marquesado }\end{array}$} \\
\hline $\begin{array}{l}\text { del Valle } \\
\text { Latifundio de } \\
\text { los Sánchez }\end{array}$ & & & 1 & & & & & 1 \\
\hline Navarros & & & 1 & & & & & 1 \\
\hline $\begin{array}{l}\text { Mayorazgos de } \\
\text { San Miguel d } \\
\text { Aguayo y de } \\
\text { San Pedro de }\end{array}$ & & & & & & & & \\
\hline Álamo & & & & & 1 & & & 1 \\
\hline $\begin{array}{l}\text { Intendencia } \\
\text { de México }\end{array}$ & & & & & & 1 & & 1 \\
\hline \multicolumn{9}{|l|}{ Obispado de } \\
\hline \multicolumn{9}{|l|}{ Arzobispado } \\
\hline de México & & & & 1 & & 1 & 1 & 3 \\
\hline $\begin{array}{l}\text { Provincia de } \\
\text { San Alberto } \\
\text { de Indias de } \\
\text { Carmelitas }\end{array}$ & & & & & & & & \\
\hline Descalzos & & & & 1 & & & & 1 \\
\hline \multicolumn{9}{|l|}{ Obispado de } \\
\hline Guadalajara & & & & & & & 1 & 1 \\
\hline \multicolumn{9}{|l|}{ Obispado } \\
\hline de Oaxaca & & & & & & & 1 & 1 \\
\hline \multicolumn{9}{|l|}{ Diódesis de } \\
\hline Oaxaca & & & & & & & 1 & 1 \\
\hline Total & 0 & 0 & 3 & 2 & 2 & 2 & 5 & 14 \\
\hline \multicolumn{9}{|c|}{ EN TORNO A ETNIAS O CULTURAS INDÍGENAS } \\
\hline Maya & 2 & & & & 1 & & & 3 \\
\hline Azteca & & 1 & & & & & & 1 \\
\hline Cora & & & 1 & & & & & 1 \\
\hline
\end{tabular}


Tabla A3c

ARTÍCULOS Y RESEÑAS EN HISTORIA MEXICANA (1951-2020). REGIONES POR CRITERIOS DE DELIMITACIÓN DE REGIONES: OTROS CRITERIOS (concluye)

\begin{tabular}{|c|c|c|c|c|c|c|c|c|}
\hline & $\begin{array}{l}1950- \\
1959\end{array}$ & $\begin{array}{r}1960- \\
1969\end{array}$ & $\begin{array}{r}1970- \\
1979\end{array}$ & $\begin{array}{r}1980- \\
1989\end{array}$ & $\begin{array}{r}1990- \\
1999\end{array}$ & $\begin{array}{l}2000- \\
2009\end{array}$ & $\begin{array}{l}2010 \\
2020\end{array}$ & Total \\
\hline \multicolumn{9}{|l|}{$\begin{array}{c}\text { Comunidades } \\
\text { nahuas y }\end{array}$} \\
\hline zapotecas & & & & & 1 & & & 1 \\
\hline Itzaes & & & & & & 1 & & 1 \\
\hline \multicolumn{9}{|l|}{ Tierras bajas } \\
\hline mayas del sur & & & & & & & 1 & 1 \\
\hline Total & 2 & 1 & 1 & 0 & 2 & 1 & 1 & 8 \\
\hline \multicolumn{9}{|l|}{ FRONTERAS } \\
\hline Frontera texana & 1 & & & & & & & 1 \\
\hline Frontera norte & 1 & & & & & & & 1 \\
\hline \multicolumn{9}{|l|}{ Frontera } \\
\hline chichimeca & & 1 & & & & & & 1 \\
\hline \multicolumn{9}{|l|}{$\begin{array}{l}\text { Frontera sureste } \\
\text { de la Nueva }\end{array}$} \\
\hline España & & & & 1 & & & & 1 \\
\hline $\begin{array}{l}\text { Frontera } \\
\text { española } \\
\text { en Norte }\end{array}$ & & & & & & & & \\
\hline América & & & & & 1 & & & 1 \\
\hline \multicolumn{9}{|l|}{ Territorios } \\
\hline de fronteras & & & & & & & 1 & 1 \\
\hline \multicolumn{9}{|l|}{ Frontera } \\
\hline Sonora- & & & & & & & & \\
\hline Arizona & & & & & & & 1 & 1 \\
\hline Total & 2 & 1 & 0 & 1 & 1 & 0 & 2 & 7 \\
\hline TOTAL REGIONES & 6 & 11 & 24 & 34 & 33 & 51 & 58 & 217 \\
\hline
\end{tabular}

FUENTE: véase la gráfica 1 . 
